\title{
Barriers to the implementation of exception reporting at a busy district general hospital
}

\author{
Authors: Dana Hassan and Fenella Maggs
}

\section{Introduction}

One change implemented with the 2016 junior doctor contract was the advent of a system of exception reporting. This enables doctors to report either loss of educational opportunities or breaches of safe working hours (including working extra hours, inability to take breaks and unsafe conditions). The outcomes of exception reporting could include compensation (with time or pay), with the process enabling unsafe working conditions to be flagged up to relevant stakeholders.

The novelty of exception reporting means that there is a paucity of data regarding uptake or improvement outcomes. One recent study from Barts Health NHS Trust explored this issue. ${ }^{1}$ The authors surveyed junior doctors within the trust and found that $35 \%$ had submitted an exception report, with $29 \%$ of respondents reporting being told not to exception report by their seniors.

Following discussions with junior doctors, it was observed that the numbers of submitted exception reports might not reflect those anticipated, given known issues with staffing levels and rota gaps.

Therefore, this study was designed to establish what percentage of eligible shifts are exception reported by junior doctors in a busy district general hospital, and the reasons behind any discrepancies in the observed pattern of exception reporting.

\section{Methods}

An online survey consisting of multiple-choice questions and free-text answers was created. This was run 'live' at two separate junior doctor teaching sessions (from foundation to core medical trainees). In addition, the survey was sent electronically to the same cohort (a total of 86 junior doctors) to enable completion outwith these sessions.

\section{Results and discussion}

There were 61 respondents in total. Ninety per cent of those surveyed did not submit all eligible exception reports, with $43 \%$ of these doctors having submitted none. Eighty-five per cent reported fear of negative repercussions by seniors as a reason for not completing reports, with $50 \%$ saying that this influenced their willingness to exception report to a moderate to significant degree. Seventy-eight per cent of respondents would be more likely to exception report if their anonymity could be maintained.

Authors: Royal United Hospital Bath

\section{Conclusion}

Low compliance is a significant barrier to implementing service improvement through exception reporting. The causes of low compliance in exception reporting are multifactorial, but discouragement from seniors and fear of repercussions was a recurrent and substantial theme.

Ensuring safe working hours is crucial to safeguarding staff wellbeing and, consequently, promoting good patient care. Exception reporting is key to promoting safe working, and therefore addressing barriers to exception reporting is critical. A number of suggestions have been identified based on this study:

> Firstly, improving the fundamentals of the exception reporting infrastructure (including addressing issues of anonymity).

> Secondly, changing supervisors' understanding of exception reporting to promote a culture of acceptance and encouragement, rather than guilt and determent.

> Thirdly, educating junior doctors on the function and system of exception reporting, as a means to providing a safe channel to raise important workplace concerns.

\section{Reference}

1 Kirwan CJ, Ali A, McCarten N. The junior doctor contract 2 years on: one trust's experience of exception reporting. $\mathrm{Br}] \mathrm{Hosp}$ Med 2018;79:640-1. 